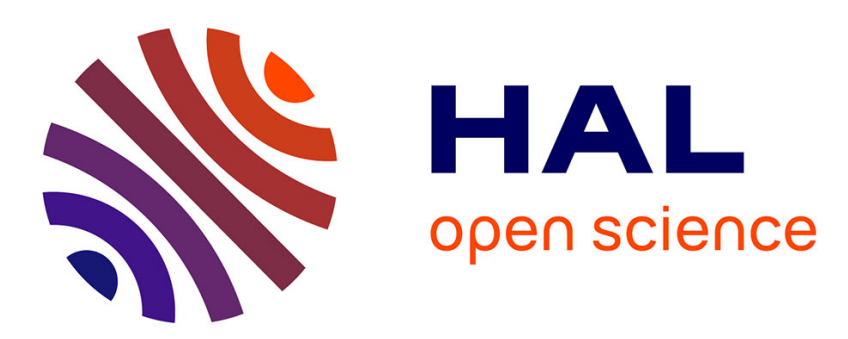

\title{
Analytical optimal solutions of impulsive out-of-plane rendezvous around elliptic orbits
}

\author{
Romain Serra, Denis Arzelier, Aude Rondepierre, Jean-Louis Calvet
}

\section{To cite this version:}

Romain Serra, Denis Arzelier, Aude Rondepierre, Jean-Louis Calvet. Analytical optimal solutions of impulsive out-of-plane rendezvous around elliptic orbits. IFAC World Congress, Aug 2014, Cape Town, South Africa. hal-01995940

\section{HAL Id: hal-01995940 \\ https://hal.science/hal-01995940}

Submitted on 28 Jan 2019

HAL is a multi-disciplinary open access archive for the deposit and dissemination of scientific research documents, whether they are published or not. The documents may come from teaching and research institutions in France or abroad, or from public or private research centers.
L'archive ouverte pluridisciplinaire HAL, est destinée au dépôt et à la diffusion de documents scientifiques de niveau recherche, publiés ou non, émanant des établissements d'enseignement et de recherche français ou étrangers, des laboratoires publics ou privés. 


\title{
Analytical optimal solutions of impulsive out-of-plane rendezvous around elliptic orbits
}

\author{
Romain Serra*, Denis Arzelier*, Aude Rondepierre** \\ Jean-Louis Calvet ${ }^{* * *}$ \\ * CNRS, LAAS, 7 avenue du colonel Roche, F-31400 Toulouse, France \\ and Univ de Toulouse, LAAS, F-31400 Toulouse, France (e-mails: \\ romain.serra@laas.fr,arzelier@laas.fr). \\ ** Institut de Mathématiques de Toulouse, Univ. de Toulouse; INSA; \\ F-31062 Toulouse, France, (email: aude.rondepierre@insa-toulouse.fr). \\ *** CNRS, LAAS, 7 avenue du colonel Roche, F-31400 Toulouse, \\ France and Univ de Toulouse, UPS, F-31400 Toulouse, France \\ (e-mail: calvet@laas.fr).
}

\begin{abstract}
This paper focuses on the fixed-time minimum-fuel out-of-plane rendezvous between close elliptic orbits of an active spacecraft, with a passive target spacecraft, assuming a linear impulsive setting, and a Keplerian relative motion. It is shown that the out-of-plane Keplerian relative dynamics are simple enough to allow for an analytical solution of the problem reviewed. The different optimal solutions, for different durations of the rendezvous, are obtained via the analysis of the optimal conditions expressed in terms of the primer vector. A numerical example illustrate sthese results.
\end{abstract}

Keywords: Impulsive optimal control, elliptic rendezvous, primer vector, analytical solution.

\section{INTRODUCTION}

For the next years, there will be an increasing demand for the efficient execution of the autonomous rendezvous between an active chaser spacecraft and a passive target spacecraft. Therefore, new challenges are met when designing appropriate guidance schemes for achieving autonomous far range rendezvous on highly elliptical orbits. Autonomy means that the simplicity of onboard implementation while preserving optimality in terms of fuel consumption, is fundamental. Here, the fixed-time linearized minimum-fuel impulsive rendezvous problem, as defined in Carter (1991), Carter and Brient (1995), is studied. The impulsive approximation for the thrust means that instantaneous velocity increments are applied to the chaser whereas its position is continuous. The focus of the paper is on the Keplerian elliptic out-of-plane rendezvous problem for which no complete solution exists at the best of our knowledge. A partial analytical solution has been given for the circular case in Carter (1991) and Prussing (1969) but as the relative motion between two vehicles in highly elliptic orbits differs significantly from the relative motion seen in circular rendezvous, the solution of the elliptic problem is much more complicated as will be seen in the sequel.

The contribution of the paper is to give a complete analytical solution of the problem whatever the duration of the rendezvous and for all possible initial and terminal conditions. These solution are obtained via the analysis of the optimal conditions expressed in terms of the primer vector as in Carter (1991) and Prussing (1969). After an- alyzing the characteristics of the dynamics of the optimal primer vector candidates, the complete analytical optimal solution is presented in every possible case. One numerical realistic example illustrates these results.

Notation: The set $\mathbb{R}_{>0}$ denotes the non-negative reals while $\mathbb{N}_{\geq 0}$ and $\mathbb{N}_{>0}$ denotes respectively the non-negative integers and the strictly positive integers. The bars $|\cdot|$ refer to the absolute value or the Euclidean norm depending whether its argument is a scalar or a matrix. sgn is the usual sign function.

\section{PROBLEM FORMULATION}

Assuming boundedness conditions on relative position and velocity, the linearized out-of-plane time-fixed fuel-optimal rendezvous problem may then be reformulated as the following optimization problem

$$
\begin{aligned}
& \min _{N, \theta_{i}, \Delta V_{i}} J=\sum_{i=1}^{N}\left|\Delta V_{i}\right| \\
& \text { s.t. } \quad z_{f}=\sum_{i=1}^{N} \frac{R\left(\theta_{i}\right)}{r\left(\theta_{i}\right)} \Delta V_{i}
\end{aligned}
$$

$N \in \mathbb{N}^{*}, \theta_{i} \in\left[\theta_{0}, \theta_{f}\right], z_{f} \in \mathbb{R}^{m}, n$ and $0 \leq e<1$ are respectively the mean motion and the eccentricity of the reference orbit. Note that the true anomaly $\theta$ has been chosen as the independent variable throughout in the paper and

$$
z_{f}=n\left(1-e^{2}\right)^{-\frac{3}{2}}\left(\phi^{-1}\left(\theta_{f}\right) \tilde{X}_{f}-\phi^{-1}\left(\theta_{0}\right) \tilde{X}_{0}\right) \neq 0
$$

$\theta_{0}$ and $\theta_{f}$ respectively denote the initial and final values of the true anomaly during the rendezvous. $\phi(\theta)$ is the 
fundamental matrix associated to the linearized relative free motion and $\Phi\left(\theta, \theta_{0}\right)=\phi(\theta) \phi^{-1}\left(\theta_{0}\right)$ denotes, therefore, the transition matrix of the linearized relative free motion. The state vectors $\tilde{X}_{f}=\tilde{X}\left(\theta_{f}\right)$ and $\tilde{X}_{0}=\tilde{X}\left(\theta_{0}\right)$ are composed of the relative positions and relative velocities vectors in the LVLH frame after the usual simplifying change of variables Yamanaka and Ankersen (2002). For the out-of-plane Keplerian elliptic rendezvous problem, we have Yamanaka and Ankersen (2002)

$$
\begin{gathered}
\phi(\theta)=\left[\begin{array}{cc}
\cos (\theta) & \sin (\theta) \\
-\sin (\theta) & \cos (\theta)
\end{array}\right] \\
R(\theta)=\left[\begin{array}{c}
-\sin (\theta) \\
\cos (\theta)
\end{array}\right], r(\theta)=1+e \cos (\theta) .
\end{gathered}
$$

The optimization decision variables are the number of impulses $N$, the sequence of thrust locations $\left\{\theta_{i}\right\}_{i=1, \cdots, N}$ and the sequence of thrusts $\left\{\Delta V_{i}\right\}_{i=1, \cdots, N}$.

\section{OPTIMALITY CONDITIONS}

If the number of impulses is fixed a priori to $N$, problem (1) may be considered as a parametric nonlinear non convex transcendental optimization problem involving the $N$ velocity increments $\Delta V\left(\theta_{i}\right)$ and $N$ locations $\theta_{i}$ of maneuvers. By applying a Lagrange multiplier rule for the problem (1) as in Carter and Brient (1995), one can derive necessary conditions of optimality (5) to (8) in terms of the Lagrange multiplier vector $\lambda \in \mathbb{R}^{m}$, as is recalled in Theorem 1 below. These conditions are also sufficient in the case of linear relative motion when strengthening them by adding the semi-infinite constraint (9) that should be fulfilled on the continuum $\left[\theta_{0}, \theta_{f}\right]$ Prussing (1995).

Theorem 1. (Lawden (1963), Neustadt (1964)).

$$
\left(\theta_{1}, \ldots, \theta_{N}, \Delta V_{1}, \ldots, \Delta V_{N}\right)
$$

is an optimal solution of problem (1) if and only if there exists a non-zero vector $\lambda \in \mathbb{R}^{m}, m=\operatorname{dim}(\phi)$ that verifies the necessary and sufficient conditions:

$$
\begin{gathered}
\Delta V_{i}=-p\left(\theta_{i}\right)\left|\Delta V_{i}\right|, \forall i=1, \cdots, N, \\
\left|\Delta V_{i}\right|=0 \text { or }\left|p\left(\theta_{i}\right)\right|=1, \forall i=1, \cdots, N, \\
\left|\Delta V_{i}\right|=0 \text { or } \theta_{i}=\theta_{0} \text { or } \theta_{i}=\theta_{f} \text { or } \\
\frac{d|p|}{d \theta}\left(\theta_{i}\right)=0, \forall i=1, \cdots, N, \\
\sum_{i=1}^{N} R\left(\theta_{i}\right) p\left(\theta_{i}\right)\left|\Delta V_{i}\right|=-z_{f}, \\
|p(\theta)| \leq 1, \quad \forall \theta \in\left[\theta_{0}, \theta_{f}\right] .
\end{gathered}
$$

where $p(\theta)$ is the so-called primer vector Carter (1991) and is defined as:

$$
p(\theta)=\frac{R(\theta)^{T} \lambda}{r(\theta)}=\frac{-\lambda_{1} \sin (\theta)+\lambda_{2} \cos (\theta)}{1+e \cos (\theta)}
$$

These results date back to the seminal work of Lawden (1963) in the early sixties, proved rigorously later by Neustadt in Neustadt (1964) and are based on the so-called primer vector theory. Obviously a primer vector candidate is completely defined by the choice of the Lagrange multipliers $\lambda_{1}, \lambda_{2}$. In the next section, the particular properties of the primer vector are analyzed such that these characteristics may be used for the derivation of the optimal solutions.

\section{PRIMER VECTOR CANDIDATE DYNAMICS}

By (10), $p(\theta)$ is obviously a $2 \pi$-periodic function. It is a harmonic oscillator weighted by the positive function $r(\theta)=1+e \cos (\theta)$. As a result, its sign changes every $\pi$. Its derivative may be computed as follows:

$$
\frac{\mathrm{d} p}{\mathrm{~d} \theta}(\theta)=-\frac{\lambda_{1}(e+\cos (\theta))+\lambda_{2} \sin (\theta)}{(1+e \cos (\theta))^{2}} .
$$

As $0 \leq e<1$, it is easy to deduce that $p(\theta)$ reaches two local extrema of opposite sign at $\theta_{e_{1}}$ and $\theta_{e_{2}}$.

\subsection{Lagrange multipliers as functions of an extremum}

If $p(\theta)$ has an extremum $p\left(\theta_{e}\right)$ at $\theta_{e}$ then it comes from (10) and (11) that:

$$
\lambda_{1}=-p\left(\theta_{e}\right) \sin \left(\theta_{e}\right), \lambda_{2}=p\left(\theta_{e}\right)\left(e+\cos \left(\theta_{e}\right)\right) .
$$

A primer vector candidate can thus be rewritten as follows:

$$
p(\theta)=p\left(\theta_{e}\right) \frac{\cos \left(\theta-\theta_{e}\right)+e \cos (\theta)}{1+e \cos (\theta)} .
$$

\subsection{Extremum ratio}

From (12) it comes that:

$$
\begin{gathered}
\frac{\left|p\left(\theta_{e_{2}}\right)\right|}{\left|p\left(\theta_{e_{1}}\right)\right|} \sin \left(\theta_{e_{2}}\right)=-\sin \left(\theta_{e_{1}}\right), \\
\frac{\left|p\left(\theta_{e_{2}}\right)\right|}{\left|p\left(\theta_{e_{1}}\right)\right|}\left(e+\cos \left(\theta_{e_{2}}\right)\right)=-\left(e+\cos \left(\theta_{e_{1}}\right)\right) .
\end{gathered}
$$

By combining equations (14) and (15), one can get a second order polynomial equation whom the ratio of the norms is a solution:

$$
X^{2}-\frac{2 e\left(e+\cos \left(\theta_{e_{1}}\right)\right)}{1-e^{2}} X-\frac{1+2 e \cos \left(\theta_{e_{1}}\right)+e^{2}}{1-e^{2}}=0 .
$$

Only the positive one of this polynomial corresponds to the ratio of norms, so that:

$$
\frac{\left|p\left(\theta_{e_{2}}\right)\right|}{\left|p\left(\theta_{e_{1}}\right)\right|}=\frac{1+2 e \cos \left(\theta_{e_{1}}\right)+e^{2}}{1-e^{2}},
$$

Note that $\left|p\left(\theta_{e_{1}}\right)\right| \neq 0$ otherwise $p(\theta) \equiv 0$ by $(12)$.

From (17), it is easily seen that the maximum norm extremum is such that $\cos \left(\theta_{e}\right) \leq-e$ whereas the minimum norm extremum is such that $\cos \left(\theta_{e}\right) \geq-e$. When $\left|p\left(\theta_{e_{1}}\right)\right|=1$ it comes that:

$$
\left|p\left(\theta_{e_{2}}\right)\right|=\frac{1+2 e \cos \left(\theta_{e_{1}}\right)+e^{2}}{1-e^{2}} .
$$

Thus $\left|p\left(\theta_{e_{2}}\right)\right|>1$ if and only if $\cos \left(\theta_{e_{1}}\right)>-e$.

\subsection{Extremum as a function of the Lagrange multipliers}

For a given $\lambda$ such that $\lambda_{2} \neq 0$, the extremum anomalies are given by the equation:

$$
\frac{\sin \left(\theta_{e}\right)}{e+\cos \left(\theta_{e}\right)}=-\frac{\lambda_{1}}{\lambda_{2}}
$$

By defining $Y=\cos \left(\theta_{e}\right)$ and $Q=\frac{\lambda_{1}}{\lambda_{2}}$, it comes after taking the square of (19) that:

$$
\left(1+Q^{2}\right) Y^{2}+2 e Q^{2} Y+e^{2} Q^{2}-1=0 .
$$


So that:

$$
\cos \left(\theta_{e}\right)=\frac{ \pm \sqrt{1+Q^{2}\left(1-e^{2}\right)}-e Q^{2}}{1+Q^{2}}
$$

and

$$
\sin \left(\theta_{e}\right)=-Q \frac{ \pm \sqrt{1+Q^{2}\left(1-e^{2}\right)}+e}{1+Q^{2}} .
$$

Thus, keeping in mind the restrictions on the maximum and minimum norm extremum, the maximum and minimum norm value of the primer vector can be expressed in terms of $\lambda$ :

$$
\begin{aligned}
& \max _{\theta \in \mathbb{R}}|p(\theta)|=\frac{\left|\lambda_{2}\right|\left(1+Q^{2}\right)}{\sqrt{1+Q^{2}\left(1-e^{2}\right)}-e}, \\
& \min _{\theta \in \mathbb{R}}|p(\theta)|=\frac{\left|\lambda_{2}\right|\left(1+Q^{2}\right)}{\sqrt{1+Q^{2}\left(1-e^{2}\right)}+e} .
\end{aligned}
$$

\section{MINIMUM-FUEL OUT-OF-PLANE OPTIMAL SOLUTIONS}

The method used to derive the analytical solution of the problem mainly consists in exploiting the different features of the optimal primer vector and in discussing all the different possible configurations of the optimal primer vector. The solution of the minimum-fuel elliptic outof-plane rendezvous problem is strongly dependent upon the duration of the rendezvous $d_{\theta}=\theta_{f}-\theta_{0}$, the initial and final anomalies $\theta_{0}$ and $\theta_{f}$ and upon the vector $z_{f}$. This dependency may be quite complicated as illustrated by the next subsections. In this section, we have tried to summarize the different solutions and the associated conditions in the most possible clearest way. For each type of optimal solution, the associated conditions involving $d_{\theta}$, $\theta_{0}, \theta_{f}$ and $z_{f}$ are given. The optimal Lagrange multipliers and related primer vector are then presented. Due to obvious space limitations, the complete derivations of the optimal solutions may be found in the reference Serra et al. (2013). Let us first define some notations that will be needed in the sequel:

$$
\begin{aligned}
& \theta_{ \pm}=\min \left\{\theta \geq \theta_{0} / \begin{array}{l}
\cos (\theta)=-e \\
\sin (\theta)= \pm \sqrt{1-e^{2}}
\end{array}\right. \\
& \theta_{i \sharp}^{ \pm}=\theta_{\sharp} \pm \arccos \left(-1-2 e \cos \left(\theta_{\sharp}\right)\right) \\
& \hat{\theta}_{i \sharp}^{ \pm}=\theta_{\sharp} \pm 2 \pi \mp \arccos \left(-1-2 e \cos \left(\theta_{\sharp}\right)\right)
\end{aligned}
$$

where $\sharp=0$ for $\theta_{i \sharp}^{+}$and $\hat{\theta}_{i \sharp}^{+}$while $\sharp=f$ for $\theta_{i \sharp}^{-}$and $\hat{\theta}_{i \sharp}^{-}$.

$$
\begin{aligned}
g^{ \pm}\left(\theta_{\sharp}\right)= & \pm e \sin \left(\theta_{\sharp}\right)+\sqrt{-e \cos \left(\theta_{\sharp}\right)\left(1+e \cos \left(\theta_{\sharp}\right)\right)} \\
& \varepsilon_{1}=\operatorname{sgn}\left(z_{f_{1}}\right), \varepsilon_{2}=\operatorname{sgn}\left(z_{f_{2}}\right), \\
& \varepsilon_{0}=\operatorname{sgn}\left(\cos \left(\theta_{0}\right) z_{f_{1}}+\sin \left(\theta_{0}\right) z_{f_{2}}\right), \\
& \varepsilon_{f}=\operatorname{sgn}\left(\cos \left(\theta_{f}\right) z_{f_{1}}+\sin \left(\theta_{f}\right) z_{f_{2}}\right) .
\end{aligned}
$$

\subsection{Two interior impulses solution}

Proposition 1. The optimal solution for the linearized impulsive out-of-plane rendezvous problem is a 2-impulse trajectory defined by the optimal locations $\theta_{ \pm}$and the defined by:

$$
\Delta V\left(\theta_{ \pm}\right)=\frac{\sqrt{1-e^{2}}}{2 e}\left(\mp e z_{f_{1}}-\sqrt{1-e^{2}} z_{f_{2}}\right),
$$

if the following conditions are verified :

$$
e\left|z_{f}\right|>\left|z_{f_{2}}\right| \text { and } d_{\theta} \geq 2 \pi
$$

or

$$
e\left|z_{f}\right|>\left|z_{f_{2}}\right| \text { and } d_{\theta}<\pi \text { and } \sin \left(\theta_{0}\right) \geq \sqrt{1-e^{2}}
$$$$
\text { and } \sin \left(\theta_{f}\right) \leq-\sqrt{1-e^{2}}
$$

or

$$
\begin{aligned}
& e\left|z_{f}\right|>\left|z_{f_{2}}\right| \text { and } d_{\theta} \geq \pi \text { and } \\
& \left\{\begin{array}{l}
\sin \left(\theta_{0}\right) \geq \sqrt{1-e^{2}} \\
\text { or } \\
\sin \left(\theta_{0}\right) \leq-\sqrt{1-e^{2}} \text { and } \sin \left(\theta_{f}\right) \leq-\sqrt{1-e^{2}} \\
\text { or } \\
\left\{\begin{array}{l}
\left|\sin \left(\theta_{0}\right)\right|<\sqrt{1-e^{2}} \text { and } \\
\left(e+\cos \left(\theta_{0}\right)\right)\left(e+\cos \left(\theta_{f}\right)\right)>0 .
\end{array}\right.
\end{array}\right.
\end{aligned}
$$

Finally, the optimal Lagrange multipliers are

$$
\begin{aligned}
& \lambda_{1}=-\varepsilon_{1} \sqrt{1-e^{2}}, \\
& \lambda_{2}=0,
\end{aligned}
$$

while the optimal primer vector is defined by:

$$
p(\theta)=\frac{\varepsilon_{1} \sqrt{1-e^{2}} \sin (\theta)}{1+e \cos (\theta)}
$$

REMARK 1. Note that when the rendezvous lasts more than $2 \pi$, the optimal solution of the planning may be chosen to be concentrated over two impulses, as is presented in Proposition 1 or spread over $N=N_{-}+N_{+}$impulses, where $N$ is defined by:

$$
\begin{aligned}
& N=N_{-}+N_{+}, \\
& N_{ \pm}=\max \left\{i \in \mathbb{N}_{>0}: \theta_{ \pm}+2(i-1) \pi \leq \theta_{f}\right\} .
\end{aligned}
$$

The optimal locations of the $N$ impulses are given by the union of the two sets:

$$
\begin{aligned}
& \left\{\theta_{i}^{+}: i=1, \cdots, N_{+}\right\} \cup\left\{\theta_{i}^{-}: i=1, \cdots, N_{-}\right\}, \\
& \theta_{i}^{ \pm}=\theta_{ \pm}+2(i-1) \pi, i=1, \cdots, N_{+} .
\end{aligned}
$$

The optimal directions of thrust are characterized by:

$$
\Delta V\left(\theta_{i}^{+}\right)=\varepsilon_{1}\left|\Delta V\left(\theta_{i}^{+}\right)\right|=-\Delta V\left(\theta_{i}^{-}\right)=\varepsilon_{1}\left|\Delta V\left(\theta_{i}^{-}\right)\right|,
$$

while the optimal amplitudes are:

$$
\begin{aligned}
& \sum_{i=1}^{N_{+}}\left|\Delta V\left(\theta_{i}^{+}\right)\right|=\varepsilon_{1} \frac{\sqrt{1-e^{2}}}{2 e}\left(e z_{f_{1}}+\sqrt{1-e^{2}} z_{f_{2}}\right), \\
& \sum_{i=1}^{N_{-}}\left|\Delta V\left(\theta_{i}^{-}\right)\right|=\varepsilon_{1} \frac{\sqrt{1-e^{2}}}{2 e}\left(e z_{f_{1}}-\sqrt{1-e^{2}} z_{f_{2}}\right) .
\end{aligned}
$$

Finally, the optimal consumption is defined by $\left|z_{f_{1}}\right| \sqrt{1-e^{2}}$.

\subsection{One interior impulse solutions}

Proposition 2. Provided that $\cos \left(\theta_{0}\right) z_{f_{1}}+\sin \left(\theta_{0}\right) z_{f_{2}} \neq 0$ and $\cos \left(\theta_{f}\right) z_{f_{1}}+\sin \left(\theta_{f}\right) z_{f_{2}} \neq 0$, the optimal solution for the linearized impulsive out-of-plane rendezvous problem is a 1-impulse trajectory defined by the optimal locations $\theta_{i *}$ :

$$
\left\{\begin{array}{l}
\cos \left(\theta_{i *}\right)=-\varepsilon_{*} \frac{z_{f_{2}}}{\left|z_{f}\right|}, \\
\sin \left(\theta_{i *}\right)=\varepsilon_{*} \frac{z_{f_{1}} \mid}{\left|z_{f}\right|},
\end{array}\right.
$$

and the optimal thrusts are defined by:

$$
\Delta V\left(\theta_{i *}\right)=-\varepsilon_{*}\left|z_{f}\right|+e z_{f_{2}},
$$

with $*=0$, if the following conditions are verified:

$$
e\left|z_{f}\right| \leq \varepsilon_{0} z_{f_{2}} \text { and } d_{\theta}<2 \pi \text { and } \varepsilon_{f}=-\varepsilon_{0}
$$


or

$e\left|z_{f}\right|>\varepsilon_{0} z_{f_{2}}$ and $d_{\theta}<2 \pi$ and $\varepsilon_{f}=-\varepsilon_{0}$

and

$\left|z_{f}\right|+\left(2 e\left|z_{f}\right|-\varepsilon_{0} z_{f_{2}}\right) \cos \left(\theta_{0}\right)+\varepsilon_{0} z_{f_{1}} \sin \left(\theta_{0}\right)>0$

and

$\varepsilon_{0}\left(e+\cos \left(\theta_{0}\right)\right) z_{f_{1}}+\left(\varepsilon_{0} z_{f_{2}}-e\left|z_{f}\right|\right) \sin \left(\theta_{0}\right)>0$

and

$\left|z_{f}\right|+\left(2 e\left|z_{f}\right|-\varepsilon_{0} z_{f_{2}}\right) \cos \left(\theta_{f}\right)+\varepsilon_{0} z_{f_{1}} \sin \left(\theta_{f}\right)>0$ and

$\varepsilon_{0}\left(e+\cos \left(\theta_{f}\right)\right) z_{f_{1}}+\left(\varepsilon_{0} z_{f_{2}}-e\left|z_{f}\right|\right) \sin \left(\theta_{f}\right)<0$

with $*=2$, if the following conditions are verified:

$$
d_{\theta} \geq 2 \pi \text { and }\left|z_{f_{2}}\right|>e\left|z_{f}\right|
$$

or

$$
\pi \leq d_{\theta}<2 \pi \text { and } e\left|z_{f}\right| \leq\left|z_{f_{2}}\right| \text { and } \varepsilon_{f}=\varepsilon_{0},
$$

or

$\pi \leq d_{\theta}<2 \pi$ and $e\left|z_{f}\right|>\left|z_{f_{2}}\right|$ and $\varepsilon_{f}=\varepsilon_{0}$ and

$\left|z_{f}\right|+\left(2 e\left|z_{f}\right|-\varepsilon_{2} z_{f_{2}}\right) \cos \left(\theta_{0}\right)+\varepsilon_{2} z_{f_{1}} \sin \left(\theta_{0}\right)>0$

and

$\varepsilon_{2} z_{f_{1}}\left(e+\cos \left(\theta_{0}\right)\right)-\left(e\left|z_{f}\right|-\varepsilon_{2} z_{f_{2}}\right) \sin \left(\theta_{0}\right)>0$

and

$\left|z_{f}\right|+\left(2 e\left|z_{f}\right|-\varepsilon_{2} z_{f_{2}}\right) \cos \left(\theta_{f}\right)+\varepsilon_{2} z_{f_{1}} \sin \left(\theta_{f}\right)>0$ and

$-\varepsilon_{2} z_{f_{1}}\left(e+\cos \left(\theta_{f}\right)\right)+\left(e\left|z_{f}\right|-\varepsilon_{2} z_{f_{2}}\right) \sin \left(\theta_{f}\right)>0$.

Finally, the optimal Lagrange multipliers are

$$
\begin{aligned}
& \lambda_{1}=-\frac{z_{f_{1}}}{\left|z_{f}\right|}, \\
& \lambda_{2}=\varepsilon_{*} e-\frac{z_{f_{2}}}{\left|z_{f}\right|},
\end{aligned}
$$

while the optimal primer vector is defined by:

$$
p(\theta)=\frac{z_{f_{1}} \sin (\theta)+\left(\varepsilon_{*} e\left|z_{f}\right|-z_{f_{2}}\right) \cos (\theta)}{(1+e \cos (\theta))\left|z_{f}\right|}
$$

REMARK 2 . When $d_{\theta} \geq 2 \pi$, as in the previous case, the optimal solution may be concentrated on one impulse or scattered in $N$ impulses defined by:

$$
N=\max \left\{i \in \mathbb{N}_{>0}: \theta_{i 2}+2(i-1) \pi \leq \theta_{f}\right\} .
$$

The optimal locations of the $N$ impulses are:

$$
\theta_{i}=\theta_{i 2}+2(i-1) \pi, i=1, \cdots, N \text {. }
$$

The optimal directions and magnitudes of thrust are then characterized by:

$$
\begin{aligned}
& \Delta V_{i}=-\varepsilon_{2}\left|\Delta V_{i}\right|, \\
& \sum_{i=1}^{N}\left|\Delta V_{i}\right|=\left|z_{f}\right|-e\left|z_{f_{2}}\right| .
\end{aligned}
$$

REMARK 3. When $\cos \left(\theta_{0}\right) z_{f_{1}}+\sin \left(\theta_{0}\right) z_{f_{2}}=0$ or $\cos \left(\theta_{f}\right) z_{f_{1}}+\sin \left(\theta_{f}\right) z_{f_{2}}=0$, the optimal solution comes down to a one impulse boundary solution for which there may exist an infinite number of optimal Lagrange multipliers. Without loss of generality, the primer vector may be chosen as in (48) where $\varepsilon_{*}=\operatorname{sgn}\left(-\sin \left(\theta_{*}\right) z_{f_{1}}+\cos \left(\theta_{*}\right) z_{f_{2}}\right)$ and $*=0$ or $*=f$.

(1) If $\cos \left(\theta_{0}\right) z_{f_{1}}+\sin \left(\theta_{0}\right) z_{f_{2}}=0$, then the optimal solution is a one initial impulse solution and the associated optimal thrust is given by:

$\Delta V\left(\theta_{0}\right)=\left(-\sin \left(\theta_{0}\right) z_{f_{1}}+\cos \left(\theta_{0}\right) z_{f_{2}}\right)\left(1+e \cos \left(\theta_{0}\right)\right)$.

(2) If $\cos \left(\theta_{f}\right) z_{f_{1}}+\sin \left(\theta_{f}\right) z_{f_{2}}=0$, then the optimal solution is a one final impulse solution and the associated optimal thrust is given by:

$\Delta V\left(\theta_{f}\right)=\left(-\sin \left(\theta_{f}\right) z_{f_{1}}+\cos \left(\theta_{f}\right) z_{f_{2}}\right)\left(1+e \cos \left(\theta_{f}\right)\right)$.

\subsection{Initial (or final) and one interior impulses}

To make the next two results clearer to the reader, we'd like to emphasize that + is associated with \# $=0$ (initial impulse) while - is associated with $\#=f$ (final impulse) as is indicated by the notation (26).

\section{Case I}

Proposition 3. The optimal solution for the linearized impulsive out-of-plane rendezvous problem is a 2-impulse trajectory defined by the optimal locations $\left(\theta_{\sharp}, \theta_{i \sharp}^{ \pm}\right)$and the associated optimal thrusts,

$$
\begin{aligned}
& \Delta V\left(\theta_{\sharp}\right)=\left(1+e \cos \left(\theta_{\sharp}\right)\right) \frac{\cos \left(\theta_{i \sharp}^{ \pm}\right) z_{f_{1}}+\sin \left(\theta_{i \sharp}^{ \pm}\right) z_{f_{2}}}{\sin \left(\theta_{i \sharp}^{ \pm}-\theta_{\sharp}\right)}, \\
& \Delta V\left(\theta_{i \sharp}^{ \pm}\right)=-\left(1+e \cos \left(\theta_{i \sharp}^{ \pm}\right)\right) \frac{\cos \left(\theta_{\sharp}\right) z_{f_{1}}+\sin \left(\theta_{\sharp}\right) z_{f_{2}}}{\sin \left(\theta_{i \sharp}^{ \pm}-\theta_{\sharp}\right)} .
\end{aligned}
$$

if the conditions:

$\sharp=0$ and $d_{\theta}<\pi$ and $\sin \left(\theta_{0}\right)<\sqrt{1-e^{2}}$

and $1+2 e \cos \left(\theta_{0}\right)+\cos \left(\theta_{f}-\theta_{0}\right) \leq 0$ and

$$
\left\{\begin{array}{l}
\varepsilon_{0}=\varepsilon_{f} \\
\text { or } \\
\varepsilon_{0}=-\varepsilon_{f} \text { and } \begin{array}{r}
\left|z_{f}\right|+\left(2 e\left|z_{f}\right|-\varepsilon_{0} z_{f_{2}}\right) \cos \left(\theta_{0}\right) \\
+\varepsilon_{0} z_{f_{1}} \sin \left(\theta_{0}\right) \leq 0
\end{array}
\end{array}\right.
$$

or

$\sharp=f$ and $d_{\theta}<\pi$ and $\sin \left(\theta_{f}\right)>-\sqrt{1-e^{2}}$

and $1+2 e \cos \left(\theta_{f}\right)+\cos \left(\theta_{f}-\theta_{0}\right) \leq 0$ and

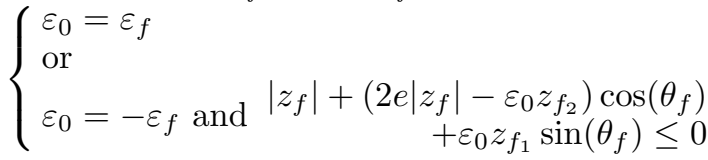

or

$\sharp=0$ and $\pi \leq d_{\theta}<2 \pi$ and

$\sin \left(\theta_{0}\right)<\sqrt{1-e^{2}}$ and $\cos \left(\theta_{0}\right) \leq 0$ and

$1-\cos \left(\theta_{f}-\theta_{0}\right)+2 \sin \left(\theta_{f}-\theta_{0}\right) g^{-}\left(\theta_{0}\right) \geq 0$

and

$\left|z_{f}\right|+\left(2 e\left|z_{f}\right|-\varepsilon_{0} z_{f_{2}}\right) \cos \left(\theta_{0}\right)+\varepsilon_{0} z_{f_{1}} \sin \left(\theta_{0}\right) \leq 0$

and

$\sin \left(\theta_{f}-\theta_{0}\right)+e\left(\sin \left(\theta_{f}\right)-\sin \left(\theta_{0}\right)\right)+$

$2\left(\cos \left(\theta_{f}-\theta_{0}\right)+e \cos \left(\theta_{0}\right)\right) g^{-}\left(\theta_{0}\right) \leq 0$

or

$\sharp=f$ and $\pi \leq d_{\theta}<2 \pi$ and

$\sin \left(\theta_{f}\right)>-\sqrt{1-e^{2}}$ and $\cos \left(\theta_{f}\right) \leq 0$ and

$1-\cos \left(\theta_{f}-\theta_{0}\right)+2 \sin \left(\theta_{f}-\theta_{0}\right) g^{+}\left(\theta_{f}\right) \geq 0$ and

$\left|z_{f}\right|+\left(2 e\left|z_{f}\right|+\varepsilon_{f} z_{f_{2}}\right) \cos \left(\theta_{f}\right)-\varepsilon_{f} z_{f_{1}} \sin \left(\theta_{f}\right) \leq 0$

and

$\sin \left(\theta_{f}-\theta_{0}\right)+e\left(\sin \left(\theta_{f}\right)-\sin \left(\theta_{0}\right)\right)+$

$2\left(\cos \left(\theta_{f}-\theta_{0}\right)+e \cos \left(\theta_{f}\right)\right) g^{+}\left(\theta_{f}\right) \geq 0$

are verified. The optimal Lagrange multipliers are given by:

$$
\begin{aligned}
\lambda_{1}= & \varepsilon_{\sharp}\left( \pm \sin \left(\theta_{\sharp}\right)\left(1+2 e \cos \left(\theta_{\sharp}\right)\right.\right. \\
& \left.-2 \cos \left(\theta_{\sharp}\right) \sqrt{-e \cos \left(\theta_{\sharp}\right)\left(1+e \cos \left(\theta_{\sharp}\right)\right)}\right), \\
\lambda_{2}= & \varepsilon_{\sharp}\left( \pm e \mp \cos \left(\theta_{\sharp}\right)\left(1+e \cos \left(\theta_{\sharp}\right)\right.\right. \\
& \left.-2 \sin \left(\theta_{\sharp}\right) \sqrt{-e \cos \left(\theta_{\sharp}\right)\left(1+2 e \cos \left(\theta_{\sharp}\right)\right)}\right),
\end{aligned}
$$

while the optimal primer vector is defined by: 


$$
p(\theta)=p\left(\theta_{i \sharp}^{ \pm}\right) \frac{\cos \left(\theta-\theta_{i \sharp}^{ \pm}\right)+e \cos (\theta)}{1+e \cos (\theta)}
$$

Case II

Proposition 4. The optimal solution for the linearized impulsive out-of-plane rendezvous problem is a 2-impulse trajectory defined by the optimal locations $\left(\theta_{\sharp}, \hat{\theta}_{i \sharp}^{ \pm}\right)$and the associated optimal thrusts given by (52), if the conditions:

$$
\begin{aligned}
& \sharp=0 \text { and } \pi \leq d_{\theta}<2 \pi \text { and } \\
& \sin \left(\theta_{0}\right)<-\sqrt{1-e^{2}} \text { and } \cos \left(\theta_{0}\right) \leq 0 \text { and } \\
& 1-\cos \left(\theta_{f}-\theta_{0}\right)-2 \sin \left(\theta_{f}-\theta_{0}\right) g^{+}\left(\theta_{0}\right) \geq 0 \\
& \text { and } \\
& 1+2 e \cos \left(\theta_{0}\right)+\cos \left(\theta_{f}-\theta_{0}\right) \geq 0 \\
& \text { and } \\
& \left|z_{f}\right|+\left(2 e\left|z_{f}\right|+\varepsilon_{0} z_{f_{2}}\right) \cos \left(\theta_{0}\right)-\varepsilon_{0} z_{f_{1}} \sin \left(\theta_{0}\right) \leq 0 \\
& \text { and } \\
& -\sin \left(\theta_{f}-\theta_{0}\right)-e\left(\sin \left(\theta_{f}\right)-\sin \left(\theta_{0}\right)\right)+ \\
& 2\left(\cos \left(\theta_{f}-\theta_{0}\right)+e \cos \left(\theta_{0}\right)\right) g^{+}\left(\theta_{0}\right) \geq 0 \\
& \sharp=f \operatorname{and} \pi \leq d_{\theta}<2 \pi \operatorname{and} \\
& \sin \left(\theta_{f}\right)>\sqrt{1-e^{2}} \text { and } \cos \left(\theta_{f}\right) \leq 0 \text { and } \\
& 1-\cos \left(\theta_{f}-\theta_{0}\right)-2 \sin \left(\theta_{f}-\theta_{0}\right) g^{-}\left(\theta_{f}\right) \geq 0 \\
& \text { and } \\
& 1+2 e \cos \left(\theta_{f}\right)+\cos \left(\theta_{f}-\theta_{0}\right) \geq 0 \\
& \text { and } \\
& \left|z_{f}\right|+\left(2 e\left|z_{f}\right|-\varepsilon_{f} z_{f_{2}}\right) \cos \left(\theta_{f}\right)+\varepsilon_{f} z_{f_{1}} \sin \left(\theta_{f}\right) \leq 0 \\
& \text { and } \\
& -\sin \left(\theta_{f}-\theta_{0}\right)-e\left(\sin \left(\theta_{f}\right)-\sin \left(\theta_{0}\right)\right)+ \\
& 2\left(\cos \left(\theta_{f}-\theta_{0}\right)+e \cos \left(\theta_{f}\right)\right) g^{-}\left(\theta_{f}\right) \geq 0
\end{aligned}
$$$$
\text { or }
$$

are verified. The optimal Lagrange multipliers are given by:

$$
\begin{aligned}
\lambda_{1}= & \varepsilon_{\sharp}\left(\mp \sin \left(\theta_{\sharp}\right)\left(1+2 e \cos \left(\theta_{\sharp}\right)\right)\right. \\
& \left.-2 \cos \left(\theta_{\sharp}\right) \sqrt{-e \cos \left(\theta_{\sharp}\right)\left(1+e \cos \left(\theta_{\sharp}\right)\right)}\right), \\
\lambda_{2}= & \varepsilon_{\sharp}\left(\mp e \pm \cos \left(\theta_{\sharp}\right)\left(1+e \cos \left(\theta_{\sharp}\right)\right)\right. \\
& \left.-2 \sin \left(\theta_{\sharp}\right) \sqrt{-e \cos \left(\theta_{\sharp}\right)\left(1+2 e \cos \left(\theta_{\sharp}\right)\right)}\right),
\end{aligned}
$$

while the optimal primer vector is defined by:

$$
p(\theta)=p\left(\hat{\theta}_{i \sharp}^{ \pm}\right) \frac{\cos \left(\theta-\hat{\theta}_{i \sharp}^{ \pm}\right)+e \cos (\theta)}{1+e \cos (\theta)}
$$

REMARK 4 . When $d_{\theta} \geq \pi$, the optimal solution may be a 3 -impulse solution made of one initial impulse, one interior impulse and one final impulse, if conditions (55)-(56), (55)(60), (56)-(59) or (58)-(62) are satisfied.

\subsection{Boundary solutions}

Proposition 5. The optimal solution for the linearized impulsive out-of-plane rendezvous problem is a 2-impulse trajectory defined by the optimal locations $\left(\theta_{0}, \theta_{f}\right)$ and the associated optimal thrusts,

$$
\begin{aligned}
& \Delta V\left(\theta_{0}\right)=\left(1+e \cos \left(\theta_{0}\right)\right) \frac{\cos \left(\theta_{f}\right) z_{f_{1}}+\sin \left(\theta_{f}\right) z_{f_{2}}}{\sin \left(\theta_{f}-\theta_{0}\right)}, \\
& \Delta V\left(\theta_{f}\right)=-\left(1+e \cos \left(\theta_{f}\right)\right) \frac{\cos \left(\theta_{0}\right) z_{f_{1}}+\sin \left(\theta_{0}\right) z_{f_{2}}}{\sin \left(\theta_{f}-\theta_{0}\right)}
\end{aligned}
$$

if the conditions

$$
\begin{aligned}
& d_{\theta}<\pi \text { and } \varepsilon_{0}=\varepsilon_{f} \text { and } \\
& \left(\left(\sin \left(\theta_{0}\right)<\sqrt{1-e^{2}}\right) \text { or }\left(\sin \left(\theta_{f}\right)>-\sqrt{1-e^{2}}\right)\right) \\
& \text { and } 1+2 e \cos \left(\theta_{f}\right)+\cos \left(\theta_{f}-\theta_{0}\right)>0 \\
& \text { and } 1+2 e \cos \left(\theta_{0}\right)+\cos \left(\theta_{f}-\theta_{0}\right)>0 \\
& \text { and } \cos \left(\theta_{f}\right) z_{f_{1}}+\sin \left(\theta_{f}\right) z_{f_{2}} \neq 0 \\
& \text { and } \cos \left(\theta_{0}\right) z_{f_{1}}+\sin \left(\theta_{0}\right) z_{f_{2}} \neq 0
\end{aligned}
$$

are verified and for which the optimal Lagrange multipliers are given by:

$$
\begin{aligned}
& \lambda_{1}=-\varepsilon_{0} \frac{\cos \left(\theta_{f}\right)+\cos \left(\theta_{0}\right)+2 e \cos \left(\theta_{0}\right) \cos \left(\theta_{f}\right)}{\sin \left(\theta_{f}-\theta_{0}\right)}, \\
& \lambda_{2}=-\varepsilon_{0} \frac{\sin \left(\theta_{f}\right)+\sin \left(\theta_{0}\right)+e \sin \left(\theta_{f}+\theta_{0}\right)}{\sin \left(\theta_{f}-\theta_{0}\right)},
\end{aligned}
$$

while the optimal primer vector is defined by:

$$
\begin{aligned}
& p(\theta)=\varepsilon_{0} \frac{\left(1+e \cos \left(\theta_{f}\right)\right) \sin \left(\theta-\theta_{0}\right)}{\sin \left(\theta_{f}-\theta_{0}\right)(1+e \cos (\theta))} \\
& -\varepsilon_{0} \frac{\sin \left(\theta_{f}-\theta\right)\left(1+e \cos \left(\theta_{0}\right)\right.}{\sin \left(\theta_{f}-\theta_{0}\right)(1+e \cos (\theta))}
\end{aligned}
$$

or

$$
\begin{gathered}
\pi<d_{\theta}<2 \pi \text { and } \varepsilon_{0}=-\varepsilon_{f} \text { and } \\
\frac{\left|\lambda_{2}\right|\left(1+Q^{2}\right)}{\sqrt{1+Q^{2}\left(1-e^{2}\right)+e}}<1
\end{gathered}
$$

are verified and for which the optimal Lagrange multipliers are given by:

$$
\begin{aligned}
& \lambda_{1}=\varepsilon_{f} \frac{\cos \left(\theta_{f}\right)-\cos \left(\theta_{0}\right)}{\sin \left(\theta_{f}-\theta_{0}\right)}, \\
& \lambda_{2}=\varepsilon_{f}\left(\frac{\sin \left(\theta_{f}\right)-\sin \left(\theta_{0}\right)}{\sin \left(\theta_{f}-\theta_{0}\right)}+e\right),
\end{aligned}
$$

while the optimal primer vector is defined by:

$$
p(\theta)=-\varepsilon_{f} \frac{\sin \left(\theta-\theta_{0}\right)+\sin \left(\theta_{f}-\theta\right)+e \cos \left(\theta_{0}\right)}{\sin \left(\theta_{f}-\theta_{0}\right)(1+e \cos (\theta))}
$$

or

$$
\begin{aligned}
& \pi<d_{\theta}<2 \pi \text { and } \varepsilon_{0}=\varepsilon_{f} \text { and } \\
& 1+2 e \cos \left(\theta_{f}\right)+\cos \left(\theta_{f}-\theta_{0}\right)<0 \text { and } \\
& 1+2 e \cos \left(\theta_{0}\right)+\cos \left(\theta_{f}-\theta_{0}\right)<0 \text { and } \\
& \cos \left(\theta_{f}\right) z_{f_{1}}+\sin \left(\theta_{f}\right) z_{f_{2}} \neq 0 \text { and } \\
& \cos \left(\theta_{0}\right) z_{f_{1}}+\sin \left(\theta_{0}\right) z_{f_{2}} \neq 0 \text { and } \\
& \left\{\begin{array}{l}
\left\{\begin{array}{l}
\left|\sin \left(\theta_{0}\right)\right|<\sqrt{1-e^{2}} \text { and } \\
\left(e+\cos \left(\theta_{0}\right)\right)\left(e+\cos \left(\theta_{f}\right)\right)<0
\end{array}\right. \\
\text { or } \\
\sin \left(\theta_{0}\right)<-\sqrt{1-e^{2}} \text { and } \sin \left(\theta_{f}\right)>-\sqrt{1-e^{2}}
\end{array}\right.
\end{aligned}
$$

are verified and for which the optimal Lagrange multipliers are given by:

$$
\begin{aligned}
& \lambda_{1}=\varepsilon_{0} \frac{\cos \left(\theta_{f}\right)+\cos \left(\theta_{0}\right)+2 e \cos \left(\theta_{0}\right) \cos \left(\theta_{f}\right)}{\sin \left(\theta_{f}-\theta_{0}\right)}, \\
& \lambda_{2}=\varepsilon_{0} \frac{\sin \left(\theta_{f}\right)+\sin \left(\theta_{0}\right)+e \sin \left(\theta_{f}+\theta_{0}\right)}{\sin \left(\theta_{f}-\theta_{0}\right)},
\end{aligned}
$$

while the optimal primer vector is defined by:

$$
\begin{aligned}
& p(\theta)=-\varepsilon_{0} \frac{\left(1+e \cos \left(\theta_{f}\right)\right) \sin \left(\theta-\theta_{0}\right)}{\sin \left(\theta_{f}-\theta_{0}\right)(1+e \cos (\theta))} \\
& +\varepsilon_{0} \frac{\sin \left(\theta_{f}-\theta\right)\left(1+e \cos \left(\theta_{0}\right)\right.}{\sin \left(\theta_{f}-\theta_{0}\right)(1+e \cos (\theta))}
\end{aligned}
$$

REMARK 5. When $d_{\theta}=\pi$ and $\theta_{0}=-\frac{\pi}{2}+k \pi, k \in \mathbb{Z}$, then the optimal solution may be concentrated on one 
boundary impulse or scattered in two boundary impulses. In that case, there may exist an infinite number of optimal Lagrange multipliers and the optimal directions and amplitudes of thrust are then characterized by:

$$
\frac{\Delta V\left(\theta_{0}\right)}{\left|\Delta V\left(\theta_{0}\right)\right|}=\varepsilon_{1} \text { and } \frac{\Delta V\left(\theta_{f}\right)}{\left|\Delta V\left(\theta_{f}\right)\right|}=-\varepsilon_{1},
$$

with $\left|\Delta V\left(\theta_{0}\right)\right|+\left|\Delta V\left(\theta_{f}\right)\right|=\left|z_{f}\right|$.

\section{NUMERICAL EXAMPLE}

The first example is based on the PROBA-3 mission whose main goals are to demonstrate the technologies required for Formation Flying of two spacecraft in highly elliptical orbit Peyrard et al. (2013). This mission is made of two independent minisatellites in HEO (Highly-elliptical Earth Orbit) in Precise Formation Flying formation. These two satellites are close to one another with the capacity to accurately control their attitude and separation. Among the different demonstrations scheduled for the PROBA3 mission, rendezvous experiments will be one of the key technologies tested for on-board autonomy. The necessary orbital elements and conditions for the out-of-plane rendezvous definition are given in Table 1.

\begin{tabular}{|c|c|}
\hline Semi-major axis & $a=37039.887 \mathrm{~km}$. \\
\hline Eccentricity & $e=0.80621$ \\
\hline$\theta_{0}$ & $2.042 \mathrm{rad}$. \\
\hline$X_{0}^{T}$ & {$\left[\begin{array}{cc}-5 & 0.5\end{array}\right] \mathrm{km} \mathrm{m} / \mathrm{s}$} \\
\hline$\theta_{f}$ & $3 \pi \mathrm{rad}$. \\
\hline$X_{f}^{T}$ & {$\left[\begin{array}{ll}20 & 0.2\end{array}\right] \mathrm{m} \mathrm{m} / \mathrm{s}$} \\
\hline
\end{tabular}

Table 1. Rendezvous parameters

The optimal solution for the impulsive rendezvous is presented in Table 2 . Note that, since $d_{\theta}>2 \pi$, it is always possible to choose an optimal solution scattered over the maximum number of impulsive maneuvers while preserving the optimal consumption as demonstrated here.

\begin{tabular}{|c|c|}
\hline$\theta_{1}(\mathrm{rad})$ & 2.5085 \\
\hline$\Delta V\left(\theta_{1}\right) \mathrm{m} / \mathrm{s}$ & -0.3492 \\
\hline$\theta_{2}(\mathrm{rad})$ & 3.7747 \\
\hline$\Delta V\left(\theta_{2}\right) \mathrm{m} / \mathrm{s}$ & 0.1639 \\
\hline$\theta_{3}(\mathrm{rad})$ & 8.7917 \\
\hline$\Delta V\left(\theta_{3}\right) \mathrm{m} / \mathrm{s}$ & -0.3492 \\
\hline Fuel Cost $\mathrm{m} / \mathrm{s}$ & 0.8624 \\
\hline
\end{tabular}

Table 2. Optimal solution for Proba 3 example

Figures 1 and 2 respectively depict the optimal out-ofplane trajectory in the phase plane and the optimal primer vector.

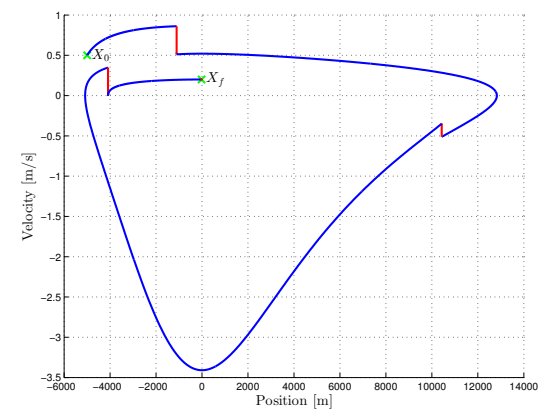

Fig. 1. Optimal trajectory in phase plane: Proba 3.



Fig. 2. Optimal primer vector: Proba 3.

\section{CONCLUSIONS}

A new analytical solution has been proposed to address the problem of time-fixed fuel-optimal out-of-plane elliptic rendezvous between spacecraft in a linear setting. Despite its apparent complexity (different number of cases and conditions), this analytical solution paves the way for onboard implementation in order to develop operational autonomy of future missions.

\section{REFERENCES}

Carter, T. (1991). Optimal impulsive space trajectories based on linear equations. Journal of Optimization Theory and Applications, 70(2). Doi: 10.1007/BF00940627.

Carter, T. and Brient, J. (1995). Linearized impulsive rendezvous problem. Journal of Optimization Theory and Applications, 86(3). Doi: 10.1007/BF02192159.

Lawden, D. (1963). Optimal trajectories for space navigation. Butterworth, London, England.

Neustadt, L. (1964). Optimization, a moment problem, and nonlinear programming. SIAM Journal of Control, 2(1), 33-53.

Peyrard, J., Escorial, D., Agenjo, A., Kron, A., and Cropp, A. (2013). Design and prototyping of proba-3 formation flying system. In Proceedings of the 5th International Conference on Spacecraft Formation Flying Missions and Technologies (SFFMT). Munich, Germany.

Prussing, J. (1969). Illustration of the primer vector in time-fixed orbit transfer. AIAA Journal, 7(6), 11671168. Doi: $10.2514 / 3.5297$.

Prussing, J. (1995). Optimal impulsive linear systems: Sufficient conditions and maximum number of impulses. Journal of the Astronautical Sciences, 43(2), 195-206.

Serra, R., Arzelier, D., Rondepierre, A., and Calvet, J. (2013). Analytical optimal solutions of impulsive outof-plane rendezvous around elliptic orbits: Results and derivations. Technical Note submitted to Journal of Guidance, Control and Dynamics 13468, LAAS-CNRS. Http://homepages.laas.fr/arzelier/publis/.

Yamanaka, K. and Ankersen, F. (2002). New state transition matrix for relative motion on an arbitrary elliptical orbit. Journal of Guidance, Control and Dynamics, 25(1). Doi: 10.2514/2.4875. 